\title{
PACKING DIMENSION OF MEAN POROUS MEASURES
}

\author{
D. BELIAEV, E. JÄRVENPÄÄ, M. JÄRVENPÄÄ, A. KÄENMÄKI, T. RAJALA, \\ S. SMIRNOV AND V. SUOMALA
}

\begin{abstract}
We prove that the packing dimension of any mean porous Radon measure on $\mathbb{R}^{d}$ may be estimated from above by a function which depends on mean porosity. The upper bound tends to $d-1$ as mean porosity tends to its maximum value. This result was stated in [BS], and in a weaker form in [JJ1, but the proofs are not correct. Quite surprisingly, it turns out that mean porous measures are not necessarily approximable by mean porous sets. We verify this by constructing an example of a mean porous measure $\mu$ on $\mathbb{R}$ such that $\mu(A)=0$ for all mean porous sets $A \subset \mathbb{R}$.
\end{abstract}

\section{INTRODUCTION}

Intuitively, it seems obvious that if a set contains relatively large holes at all small scales then the dimension of the set should be smaller than that of the ambient space. This observation was generalized and made into a quantitative form by Mattila [Ma1] in terms of a concept called porosity which describes the sizes of holes at all small scales (for the definition see Section 2). Mattila proved that if the porosity of a subset of $\mathbb{R}^{d}$ is close to its maximum value $\frac{1}{2}$ then its Hausdorff dimension cannot be much bigger than $d-1$. The correct asymptotic behaviour was established by Salli [S]. He also showed that Hausdorff dimension may be replaced by packing dimension, and moreover, by box counting dimension under the assumption that the set is uniformly porous.

The above mentioned result for Hausdorff dimension fails if the set contains large holes only at sequences of arbitrarily small scales; there are examples of such sets in $\mathbb{R}^{d}$ with Hausdorff dimension $d$ [Ma2]. Nevertheless, the assumption that the set has relatively large holes at all small scales may be weakened to obtain an upper bound for Hausdorff dimension, or more generally, for packing dimension. In fact, it is sufficient to suppose that a certain percentage of scales

2000 Mathematics Subject Classification. 28A75, 28A80.

Key words and phrases. Porosity, mean porosity, packing dimension, Radon measures.

EJ, MJ, AK, TR and VS acknowledge the support of the Academy of Finland (projects \# 211229 and \# 114821) and the Centre of Excellence in Analysis and Dynamics Research. EJ also thanks the hospitality of the University of Geneva. DB, EJ and SS acknowledge the support of the Swiss National Science Foundation. TR appreciates the financial support of Vilho, Yrjö and Kalle Väisälä foundation. Finally, we all thank the referee for valuable comments clarifying the exposition. 
contains holes. This leads to the concept of mean porosity (for the definition see Section 2). Dimensional properties of such sets were considered by Koskela and Rohde [KR] in the case of small mean porosity, and by Beliaev and Smirnov in the case of large one. For other related results, see [JJKS.

In this paper, the emphasis is given to packing dimensions of mean porous measures (for the definition see Section 21). Porous measures were introduced by Eckmann, Järvenpää and Järvenpää [EJJ] whilst the analogue of Mattila's result was verified for porous measures in [JJ1]. Note that in [JJ1] the results are claimed for packing dimension but the argument works only for Hausdorff dimension as explained in [JJ2].

The study of mean porous measures was pioneered by Beliaev and Smirnov [BS]. In [BS the proof of the statement that the same upper bound which is valid for packing dimensions of mean porous sets holds for mean porous measures as well is based on a proposition claiming that mean porous measures are approximable by mean porous sets. However, this is not the case: in Theorem 4.1 we construct a mean porous measure $\mu$ such that all mean porous sets have zero $\mu$-measure. The main purpose of this paper is to develop a new method to show that the statements of [BS, [JJ1] are true (see Theorem [3.1) even though the proofs are not correct.

The paper is organized as follows: In Section 2 we discuss the basic concepts. Section 3 is dedicated to the proof of our main result. Besides this, we illustrate by an example that the upper bound which we obtain is asymptotically the best possible one. Finally, in the last section we construct an example of a mean porous measure which is not approximable by mean porous sets.

\section{BASIC CONCEPTS}

In this section, we give the basic definitions used throughout the paper. Intuitively, the porosity of a set gives for all small scales the relative radius of the largest ball which fits into a reference ball centered at the set and which does not intersect the set. Let $A \subset \mathbb{R}^{d}$. For all $x \in \mathbb{R}^{d}$ and $r>0$, we define

$$
\operatorname{por}(A, x, r)=\sup \left\{\alpha \geq 0: B(y, \alpha r) \subset B(x, r) \backslash A \text { for some } y \in \mathbb{R}^{d}\right\} \text {. }
$$

Here $B(x, r)$ is the closed ball with centre at $x$ and radius $r$. Clearly, $0 \leq$ $\operatorname{por}(A, x, r) \leq \frac{1}{2}$ for all $x \in A$. Given $0 \leq \alpha \leq \frac{1}{2}$, the set $A$ is said to be $\alpha$-porous at $x$ if

$$
\liminf _{r \rightarrow 0} \operatorname{por}(A, x, r) \geq \alpha .
$$

Moreover, $A$ is $\alpha$-porous if it is $\alpha$-porous at every point $x \in A$.

For measures, the corresponding concepts are defined as follows: Let $\mu$ be a Radon measure on $\mathbb{R}^{d}$. For all $x \in \mathbb{R}^{d}$ and for all positive real numbers $r$ and $\varepsilon$, 
set

$$
\begin{aligned}
\operatorname{por}(\mu, x, r, \varepsilon)= & \sup \left\{\alpha \geq 0: \text { there is } z \in \mathbb{R}^{d}\right. \text { such that } \\
& B(z, \alpha r) \subset B(x, r) \text { and } \mu(B(z, \alpha r)) \leq \varepsilon \mu(B(x, r))\} .
\end{aligned}
$$

Given $\alpha \geq 0$, the measure $\mu$ is $\alpha$-porous at a point $x \in \mathbb{R}^{d}$ if

$$
\lim _{\varepsilon \rightarrow 0} \liminf _{r \rightarrow 0} \operatorname{por}(\mu, x, r, \varepsilon) \geq \alpha .
$$

The order of taking limits is important here: if we changed it we would obtain the porosity of spt $\mu$, the support of $\mu$. Finally, the measure $\mu$ is $\alpha$-porous if there is $A \subset \mathbb{R}^{d}$ with $\mu(A)>0$ such that $\mu$ is $\alpha$-porous at every point $x \in A$. It is not difficult to see that in this case $0 \leq \alpha \leq \frac{1}{2}$. For more information on porosity of measures, see [EJJ].

Larger classes of mean porous sets and measures are obtained by demanding that a certain percentage of scales - not necessarily all small ones - are porous. Given $\alpha \geq 0$ and a positive integer $j$, the set $A$ is $\alpha$-porous for scale $j$ at a point $x \in \mathbb{R}^{d}$ whenever $\operatorname{por}\left(A, x, 2^{-j}\right) \geq \alpha$. For $0<p \leq 1$, the set $A$ is called mean $(\alpha, p)$-porous at a point $x \in \mathbb{R}^{d}$ if

$$
\liminf _{i \rightarrow \infty} \frac{\#\left\{1 \leq j \leq i: \operatorname{por}\left(A, x, 2^{-j}\right) \geq \alpha\right\}}{i} \geq p .
$$

Here the cardinality of a set is denoted by \#. We say that $A$ is mean $(\alpha, p)$ porous if it is mean $(\alpha, p)$-porous at every point $x \in A$. The measure $\mu$, in turn, is mean $(\alpha, p)$-porous at $x$ if

$$
\lim _{\varepsilon \rightarrow 0} \liminf _{i \rightarrow \infty} \frac{\#\left\{1 \leq j \leq i: \operatorname{por}\left(\mu, x, 2^{-j}, \varepsilon\right) \geq \alpha\right\}}{i} \geq p .
$$

Finally, $\mu$ is mean $(\alpha, p)$-porous if there is $A \subset \mathbb{R}^{d}$ with $\mu(A)>0$ such that $\mu$ is mean $(\alpha, p)$-porous at all points $x \in A$.

The packing dimension, $\operatorname{dim}_{\mathrm{p}}$, of a Radon measure $\mu$ on $\mathbb{R}^{d}$ is defined in terms of local dimensions as follows:

$$
\operatorname{dim}_{\mathrm{p}} \mu=\mu \text { - } \operatorname{ess}_{x \in \mathbb{R}^{d}} \overline{\operatorname{dim}}_{\mathrm{loc}} \mu(x)
$$

where

$$
\overline{\operatorname{dim}}_{\text {loc }} \mu(x)=\limsup _{r \downarrow 0} \frac{\log (\mu(B(x, r)))}{\log r}
$$

and $\mu$-ess inf means the essential infimum with respect to $\mu$. Equivalently, the packing dimension of $\mu$ is given by means of packing dimensions of Borel sets with positive $\mu$-measure [C]:

$$
\operatorname{dim}_{\mathrm{p}} \mu=\inf \left\{\operatorname{dim}_{\mathrm{p}} A: A \text { is a Borel set with } \mu(A)>0\right\} .
$$


Remark 2.1. Replacing the essential infimum by the essential supremum in the above definition leads to the concept of upper packing dimension. Using the fact that restricting a measure will not decrease the porosity [EJJ], we see that Theorem 3.1 is valid for the upper packing dimension of $\mu$ as well provided that $\mu$ is mean porous $\mu$-almost everywhere.

\section{PACKING Dimension of MEASURES With LARGE MEAN POROSITY}

In this section we prove the following packing dimension estimate for mean porous measures:

Theorem 3.1. Let $0 \leq \alpha \leq \frac{1}{2}$ and $0<p \leq 1$. There exists a constant $C$ depending only on $d$ such that for all mean $(\alpha, p)$-porous Radon measures $\mu$ on $\mathbb{R}^{d}$ we have

$$
\operatorname{dim}_{\mathrm{p}} \mu \leq d-p+\frac{C}{\log \left(\frac{1}{1-2 \alpha}\right)} .
$$

At the end of this section, we give a construction (Example 3.9) which indicates that the upper bound of Theorem 3.1 is asymptotically the best possible one as $\alpha$ tends to $\frac{1}{2}$. The proof of Theorem 3.1 is given as a series of lemmas. The first one serves as a key tool in the proof of our main result. We use the symbol $r_{Q}$ for the side-length of a cube $Q \subset \mathbb{R}^{d}$.

Lemma 3.2. Let $m, i_{0} \in \mathbb{N}$ and $D>0$. Let $\mu$ be a Radon measure on $\mathbb{R}^{d}$ with $0<\mu\left(\mathbb{R}^{d}\right)<\infty$. Assume that all disjoint collections $\mathcal{Q}$ of half-open $2^{m}$-adic cubes with side-length at most $2^{-m i_{0}}$ have the following property: for all $Q \in \mathcal{Q}$ there is $0<\tau(Q)<D$ such that

$$
\sum_{Q \in \mathcal{Q}} r_{Q}^{\tau(Q)} \mu(Q)^{1-\frac{\tau(Q)}{D}}<\mu\left(\mathbb{R}^{d}\right)
$$

Then

$$
\operatorname{dim}_{\mathrm{p}} \mu \leq D
$$

Proof. Suppose to the contrary that $\operatorname{dim}_{\mathrm{p}} \mu>D$. Then $\overline{\operatorname{dim}}_{\text {loc }} \mu(x)>D$ for $\mu$-almost every $x \in \mathbb{R}^{d}$. Recall from [C, Lemma 2.3] that for $\mu$-almost all $x \in \mathbb{R}^{d}$ the local dimension $\overline{\operatorname{dim}}_{\text {loc }} \mu(x)$ may be calculated using half-open $2^{m}$-adic cubes containing $x$ instead of balls $B(x, r)$. Hence, for $\mu$-almost every $x \in \mathbb{R}^{d}$ we may choose a cube $Q_{x}$ containing $x$ and with side-length $2^{-m i}$ for some $i>i_{0}$ such that $\mu\left(Q_{x}\right)<r_{Q_{x}}^{D}$. Let $\mathcal{Q}$ be a disjoint collection of such cubes covering $\mu$-almost all points of $\mathbb{R}^{d}$. Then

$$
\mu\left(\mathbb{R}^{d}\right)=\sum_{Q \in \mathcal{Q}} \mu(Q)^{\frac{\tau(Q)}{D}} \mu(Q)^{1-\frac{\tau(Q)}{D}}<\sum_{Q \in \mathcal{Q}} r_{Q}^{\tau(Q)} \mu(Q)^{1-\frac{\tau(Q)}{D}}<\mu\left(\mathbb{R}^{d}\right),
$$

which is a contradiction. 
The following lemma shows that when cubes $Q$ are of the same size we can approximate the sums $\sum_{Q} r_{Q}^{\tau} \mu(Q)^{1-\frac{\tau}{D}}$ from above by distributing the measure evenly on the cubes.

Lemma 3.3. Let $D>0$. Assume that $0<\tau<D$ and $Q^{1}, \ldots, Q^{N} \subset \mathbb{R}^{d}$ are disjoint cubes with side-length $r$. Then for any Radon measure $\mu$ on $\mathbb{R}^{d}$

$$
\left.\sum_{j=1}^{N} r^{\tau} \mu\left(Q^{j}\right)^{1-\frac{\tau}{D}} \leq N^{\frac{\tau}{D}} r^{\tau} \mu\left(\bigcup_{j=1}^{N} Q^{j}\right)\right)^{1-\frac{\tau}{D}}\left(=N r^{\tau}\left(\frac{\mu\left(\bigcup_{j=1}^{N} Q^{j}\right)}{N}\right)^{1-\frac{\tau}{D}}\right) .
$$

Proof. Since the cubes $Q^{1}, \ldots, Q^{N}$ are of the same size, the claim follows directly from Hölder's inequality:

$$
\sum_{j=1}^{N} r^{\tau} \mu\left(Q^{j}\right)^{1-\frac{\tau}{D}}=r^{\tau} \sum_{j=1}^{N} \mu\left(Q^{j}\right)^{1-\frac{\tau}{D}} \leq N^{\frac{\tau}{D}} r^{\tau}\left(\sum_{j=1}^{N} \mu\left(Q^{j}\right)\right)^{1-\frac{\tau}{D}} .
$$

The proof of the next lemma is based on straightforward geometric arguments. The boundary of a set $A \subset \mathbb{R}^{d}$ is denoted by $\partial A$.

Lemma 3.4. Let $k \in \mathbb{N}$ and let $Q \subset \mathbb{R}^{d}$ be a dyadic cube. If $B_{1}, B_{2}, \ldots B_{n}$ are closed balls with radii at least $\sqrt{d} r_{Q}$, then $\partial\left(Q \backslash \bigcup_{i=1}^{n} B_{i}\right)$ may be covered by $c 2^{k(d-1)}$ dyadic cubes of side length $2^{-k} r_{Q}$. Here $c=c(d)$ is a positive and finite constant depending only on $d$.

Proof. Let $B_{i}=B\left(x_{i}, r_{i}\right)$ and $\Gamma=\partial\left(Q \backslash \bigcup_{i=1}^{n} B_{i}\right)$. We may assume that $B_{i} \cap Q \neq \emptyset$ and $x_{i} \notin Q$ since $r_{i} \geq \sqrt{d} r_{Q}$. Denote the faces of $Q$ by $F_{1}, \ldots, F_{2 d}$, and divide the balls $B_{i}$ into $2 d$ disjoint sets $A_{j}$ such that $B_{i} \in A_{j}$ provided $F_{j}$ is the closest face to $x_{i}$. Here the distance is measured from the centre of a face. (If there are several faces which are equally close choose one of those.) Fix $j \in\{1, \ldots, 2 d\}$ and consider the part of $\Gamma$ determined by $A_{j}$. Since $r_{i} \geq \sqrt{d} r_{Q}$ we have that $F_{j} \subset B_{i}$ if $x_{i}$ is sufficiently close to $F_{j}$. Hence, there is a constant $\gamma>0$ such that the angle between the normal of $F_{j}$ and the tangent plane of $B_{i}$ is larger than $\gamma$ at any point in $\Gamma \cap B_{i}$ for all $B_{i} \in A_{j}$. The minimum point for this angle is obtained in the following manner: Consider a vertex $v$ of $Q$ contained in $F_{j}$. Denote by $v^{\prime}$ the vertex of $F_{j}$ which is opposite to $v$, that is, the line segment determined by $v$ and $v^{\prime}$ is a diagonal of $F_{j}$. Let $L$ be the line determined by the diagonal of $Q$ containing $v$. Move along $L$ away from $Q$ up to the point $y$ where the distance to $v^{\prime}$ equals $\sqrt{d} r_{Q}$. Now $y$ is a minimum point which determines $\gamma$. We conclude that there is a bi-Lipschitz injection from $\cup_{B_{i} \in A_{j}} B_{i} \cap \Gamma$ to $F_{j}$ such that the Lipschitz constants depend only on $\gamma$. This gives the claim.

Before stating the rest of the auxiliary results, we introduce the notation we need throughout the remaining part of this section. 
Consider the smallest integer $l$ such that $4 \sqrt{d} \leq 2^{l}$. For $\frac{15}{32}<\alpha<\frac{1}{2}$, let $k=k(\alpha)$ be the unique positive integer for which

$$
\sqrt{d} 2^{-k-1} \leq(1-2 \alpha) 2^{l}<\sqrt{d} 2^{-k}
$$

For all $i \in \mathbb{N}$, the collection of all half-open $2^{k}$-adic cubes of side-length $2^{-k i}$ is denoted by $\mathcal{Q}^{i}$. Moreover, if $Q \in \mathcal{Q}^{i}$ and $Q^{\prime} \in \mathcal{Q}^{i+n}$, we use the notation $Q^{\prime} \prec_{n} Q$ provided that $Q^{\prime} \subset Q$. This is simplified to $Q^{\prime} \prec Q$ in the case $n=1$.

Let $\mu$ be a Radon measure on $\mathbb{R}^{d}$. Given $\varepsilon>0$, we call a cube $Q \in \mathcal{Q}^{i+1}$ porous provided that

$$
\operatorname{por}\left(\mu, x, 2^{-k i+l}, \varepsilon\right) \geq \alpha
$$

for some $x \in Q$. For all $Q \in \mathcal{Q}^{i}$, set

$$
Q_{\text {por }}=\bigcup\left\{Q^{\prime} \prec Q: Q^{\prime} \text { is porous }\right\} \text {. }
$$

Finally, for $x \in \mathbb{R}^{d}$ let $Q_{x}^{i} \in \mathcal{Q}^{i}$ be the unique cube containing $x$. If $c>0, c Q$ is the cube obtained from a cube $Q$ by magnifying by the factor $c$ with respect to the centre of $Q$.

One of the fundamental and most useful structural properties of porous sets is the following: if the porosity of $A \subset \mathbb{R}^{d}$ is close to $\frac{1}{2}$, then locally inside each ball with radius $r$ the set $A$ is contained in a small neighbourhood of some $(d-1)$ dimensional surface with $\mathcal{H}^{d-1}$-measure comparable to $r^{d-1}$. For more precise statements of this type, see e.g. [S] or [JJKS]. In the following lemma which is a slight improvement of [JJ1, Lemma 2.2] we translate this fact into the language of mean porous measures. It states that every cube $Q$ may be divided into three parts $Q=E \cup P \cup J$, where $E$ has small measure, $P$ is a small neighbourhood of some $(d-1)$-dimensional surface and $J$ contains no porous points.

Lemma 3.5. Let $\mu$ be a Radon measure on $\mathbb{R}^{d}, i \in \mathbb{N}$ and $\varepsilon>0$. Then any cube $Q \in \mathcal{Q}^{i}$ may be divided into three parts

$$
Q=E \cup P \cup J,
$$

where $\mu(E) \leq N \varepsilon \mu\left(\left(1+2^{l+1}\right) Q\right), J \cap Q_{\text {por }}=\emptyset$, and $P$ can be covered by at most $c 2^{k(d-1)}$ cubes $Q^{\prime} \prec Q$. Here $N=N(\alpha, d)$ and $c=c(d)$ are positive and finite constants.

Proof. For any $Q^{\prime} \prec Q$ with $Q^{\prime} \subset Q_{\text {por }}$, there is $x \in Q^{\prime}$ such that

$$
\operatorname{por}\left(\mu, x, 2^{-k i+l}, \varepsilon\right) \geq \alpha \text {. }
$$

Hence, given $0<\alpha^{\prime}<\alpha$ with $\left(1-2 \alpha^{\prime}\right) 2^{l}<\sqrt{d} 2^{-k}$, we find a ball $B_{Q^{\prime}}$ of radius $\alpha^{\prime} 2^{-k i+l}$ such that

$$
B_{Q^{\prime}} \subset B\left(x, 2^{-k i+l}\right) \subset\left(1+2^{l+1}\right) Q \text { and } \mu\left(B_{Q^{\prime}}\right) \leq \varepsilon \mu\left(\left(1+2^{l+1}\right) Q\right) .
$$


Denoting by $\operatorname{dist}(a, A)$ the distance from a point $a \in \mathbb{R}^{d}$ to a set $A \subset \mathbb{R}^{d}$, define

$$
\begin{aligned}
E & =\bigcup_{\substack{Q^{\prime} \prec Q, Q^{\prime} \subset Q_{\text {por }}}} B_{Q^{\prime}} \cap Q, \\
P & =\left\{x \in Q: \operatorname{dist}(x, \partial(Q \backslash E))<2^{-k(i+1)+l}\right\}, \text { and } \\
J & =Q \backslash(E \cup P) .
\end{aligned}
$$

It is evident from (3.2) that

$$
\mu(E) \leq 2^{k d} \varepsilon \mu\left(\left(1+2^{l+1}\right) Q\right)=N(\alpha, d) \varepsilon \mu\left(\left(1+2^{l+1}\right) Q\right) .
$$

On the other hand, since $\alpha^{\prime}>\frac{7}{16}$, we have that $\alpha^{\prime} 2^{-k i+l}>\sqrt{d} r_{Q}$, and so it follows easily from Lemma 3.4 that $P$ may be covered by at most $\left(1+2^{l+1}\right)^{d} c^{\prime} 2^{k(d-1)}=$ $c(d) 2^{k(d-1)}$ cubes $Q^{\prime} \prec Q$ where $c^{\prime}=c^{\prime}(d)$ is the constant of Lemma 3.4.

It remains to show that $J \cap Q_{\text {por }}=\emptyset$, that is,

$$
Q_{\text {por }} \subset E \cup P \text {. }
$$

Consider $y \in Q_{\text {por }} \backslash E$ and choose $Q^{\prime} \prec Q$ with $y \in Q^{\prime} \subset Q_{\text {por }}$. Let $x \in Q^{\prime}$ and $B_{Q^{\prime}}$ be as in (3.2). By the choice of $\alpha^{\prime}$ we have

$$
\operatorname{dist}\left(y, B_{Q^{\prime}}\right) \leq|y-x|+\left(1-2 \alpha^{\prime}\right) 2^{-k i+l}<2 \sqrt{d} 2^{-k(i+1)} \leq 2^{-k(i+1)+l}
$$

which gives $\operatorname{dist}(y, \partial(Q \backslash E))<2^{-k(i+1)+l}$, and therefore $y \in P$. This completes the proof of (3.3) .

Next lemma is in the core of the proof of Theorem 3.1. It shows that summing over porous subcubes of a cube $Q$ gives a small factor which is decreasing exponentially under iteration. This is needed when proving that the assumptions of Lemma 3.2 are valid.

For the purpose of formulating our key lemma, we define weights $\beta(Q)$ for cubes $Q \in \mathcal{Q}^{i}$ as follows: Suppose that $\mu$ is a Radon measure on $\mathbb{R}^{d}$ and $0<D<d$. Set

$$
C=C(d)=\max \left\{c, 2 d 2^{l}\right\}
$$

where $c=c(d)$ is as in Lemma 3.5. If $Q \in \mathcal{Q}^{i}$ for some $i \in \mathbb{N}$, define

$$
\beta(Q)=\frac{1}{3} \begin{cases}C^{-\frac{1}{2}} 2^{-\frac{k}{2}(d-1-D)} & \text { if } Q \text { is porous } \\ 2^{-\frac{k}{2}(d-D)} & \text { otherwise. }\end{cases}
$$

For all $n \in \mathbb{N}$ with $n \geq 2$, let $\varepsilon_{0}=\varepsilon_{0}(d, \alpha, D, n)>0$ be the unique real number satisfying

$$
R\left(\varepsilon_{0}\right)=\frac{5}{18} C^{-\frac{1}{2}} 2^{\frac{k}{2}}
$$


where for all $\varepsilon>0$

$$
\begin{aligned}
& R(\varepsilon)=R(\varepsilon, n)= \\
& \frac{n-1}{3}(\varepsilon N)^{\frac{1}{2}} C^{-\frac{1}{2}} 2^{\frac{k}{2}} 2^{k(n-1)\left(d-\frac{D}{2}\right)} \max \left\{1, \frac{1}{3} C^{-\frac{1}{2}} 2^{-\frac{k}{2}(d-1-D)}\right\}^{n-1}
\end{aligned}
$$

and $N=N(\alpha, d)$ is as in Lemma 3.5 .

Before formulating our lemma we make one more remark: From now on, we assume that $\frac{15}{32}<\alpha<\frac{1}{2}$ is so close to $\frac{1}{2}$ that

$$
k=k(\alpha) \geq \frac{\log C}{\log 2} .
$$

Thus $C^{-\frac{1}{2}} 2^{-\frac{k}{2}(d-1-D)} \geq 2^{-\frac{k}{2}(d-D)}$, giving

$$
\beta(Q) \leq \frac{1}{3} C^{-\frac{1}{2}} 2^{-\frac{k}{2}(d-1-D)}
$$

for all $Q$. This fact will be used repeatedly in the proof of Lemma 3.6.

For any $Q \in \mathcal{Q}^{i}$ and $j=0,1,2, \ldots, i$, we denote by $Q_{j}$ the unique cube in $\mathcal{Q}^{j}$ for which $Q \subset Q_{j}$. Clearly, $Q_{i} \subset Q_{i-1} \subset \cdots \subset Q_{1} \subset Q_{0}$ and $Q_{i}=Q$.

Lemma 3.6. Let $\mu$ be a Radon measure on $\mathbb{R}^{d}, 0<D<d$ and $n \in \mathbb{N}$ with $n \geq 2$. Then for all $i \in \mathbb{N}$ and $Q \in \mathcal{Q}_{i}$ we have

$$
\sum_{Q^{\prime} \prec n Q}\left(\prod_{j=1}^{n} \beta\left(Q_{i+j}^{\prime}\right)\right) r_{Q^{\prime}}^{\frac{D}{2}} \mu\left(Q^{\prime}\right)^{\frac{1}{2}} \leq C^{-\frac{1}{2}} 2^{\frac{k}{2}} r_{Q}^{\frac{D}{2}} \mu(Q)^{\frac{1}{2}} .
$$

Moreover, if $\mathcal{Q}$ is any finite collection of disjoint $2^{k n}$-adic cubes and $\mu$ is a Radon measure on $\mathbb{R}^{d}$ such that spt $\mu \subset[0,1]^{d}$, then

$$
\sum_{Q \in \mathcal{Q}}\left(\prod_{j=0}^{N_{Q}-1} C^{\frac{1}{2}} 2^{-\frac{k}{2}} \prod_{i=j n+1}^{(j+1) n} \beta\left(Q_{i}\right)\right) r_{Q}^{\frac{D}{2}} \mu(Q)^{\frac{1}{2}} \leq r_{[0,1]^{d}}^{\frac{D}{2}} \mu\left(\mathbb{R}^{d}\right)^{\frac{1}{2}}
$$

where $N_{Q} \in \mathbb{N}$ such that $r_{Q}=2^{-k n N_{Q}}$.

Proof. The proof is based on Lemma 3.5. The problematic part in Lemma 3.5 is the factor $\mu\left(\left(1+2^{l+1}\right) Q^{\prime}\right)$. If $Q^{\prime}$ is close to the boundary of $Q$ the expanded cube $\left(1+2^{l+1}\right) Q^{\prime}$ will not be a subset of $Q$, and therefore we are unable to estimate the sum in terms of $\mu$-measure of $Q$. This problem is overcome by dividing the subcubes of $Q$ into two parts depending on there distance to the boundary of $Q$. This leads to the use of $n$ : the larger $n$ we take, the better estimates we have. For $n=1$ we cannot utilize porosity at all.

Claim (3.11) is a direct consequence of repeated applications of (3.10). In what follows we will prove (3.10) by induction. Divide the cube $Q \in \mathcal{Q}^{i}$ into two regions of subcubes, the boundary region $\mathcal{Q}_{B} \subset \mathcal{Q}^{i+1}$ and the interior one $\mathcal{Q}_{I} \subset \mathcal{Q}^{i+1}$, as follows

$$
\mathcal{Q}_{B}=\left\{Q^{\prime} \prec Q: \operatorname{dist}\left(Q^{\prime}, \partial Q\right) \leq 2^{-(i+1) k+l}\right\}
$$


and

$$
\mathcal{Q}_{I}=\left\{Q^{\prime} \prec Q: \operatorname{dist}\left(Q^{\prime}, \partial Q\right)>2^{-(i+1) k+l}\right\}
$$

For $n=2$, we estimate the sum in these subcubes in the following manner: Let $R(\varepsilon)=R(\varepsilon, 2)$ and $\varepsilon_{0}$ be as in (3.6) and (3.7). Take $Q^{\prime \prime} \in \mathcal{Q}_{I}$. Defining $Q_{\text {por }}^{\prime \prime}$ in terms of $\varepsilon_{0}$, we obtain from definition (3.5) and Lemma 3.3 that

$$
\begin{aligned}
& \sum_{\substack{Q^{\prime} \prec Q^{\prime \prime} \\
Q^{\prime} \cap Q_{p o r}^{\prime \prime}=\emptyset}} \beta\left(Q^{\prime}\right) r_{Q^{\prime}}^{\frac{D}{2}} \mu\left(Q^{\prime}\right)^{\frac{1}{2}} \\
\leq & \frac{1}{3} 2^{-\frac{k}{2}(d-D)} 2^{\frac{k d}{2}} 2^{-\frac{k}{2}(i+2) D} \mu\left(Q^{\prime \prime}\right)^{\frac{1}{2}} \\
= & \frac{1}{3} 2^{-\frac{k}{2}(i+1) D} \mu\left(Q^{\prime \prime}\right)^{\frac{1}{2}} .
\end{aligned}
$$

To estimate the sum over $Q^{\prime} \subset Q_{\text {por }}^{\prime \prime}$, we apply Lemma 3.5 to $Q^{\prime \prime}$. Note that the part $J$ is now absent, and therefore the sum is divided into two parts determined by $E$ and $P$. Using Lemma 3.3 in both parts and the fact that $\left(1+2^{l+1}\right) Q^{\prime \prime} \subset Q$, we have recalling (3.4)

$$
\begin{aligned}
& \sum_{\substack{Q^{\prime} \prec Q^{\prime \prime} \\
Q^{\prime} \subset Q_{p o r}^{\prime \prime}}} \beta\left(Q^{\prime}\right) r_{Q^{\prime}}^{\frac{D}{2}} \mu\left(Q^{\prime}\right)^{\frac{1}{2}} \\
\leq & \sum_{\substack{Q^{\prime} \prec Q^{\prime \prime} \\
Q^{\prime} \subset Q_{p o r}^{\prime \prime}, Q^{\prime} \cap E \neq \emptyset}} \beta\left(Q^{\prime}\right) r_{Q^{\prime}}^{\frac{D}{2}} \mu\left(Q^{\prime}\right)^{\frac{1}{2}}+\sum_{\substack{Q^{\prime} \prec Q^{\prime \prime} \\
Q^{\prime} \subset Q_{p o r}^{\prime \prime}, Q^{\prime} \cap P \neq \emptyset}} \beta\left(Q^{\prime}\right) r_{Q^{\prime}}^{\frac{D}{2}} \mu\left(Q^{\prime}\right)^{\frac{1}{2}} \\
\leq & \frac{1}{3} C^{-\frac{1}{2}} 2^{-\frac{k}{2}(d-1-D)}\left(2^{\frac{k d}{2}} 2^{-\frac{k}{2}(i+2) D}\left(N \varepsilon_{0} \mu\left(\left(1+2^{l+1}\right) Q^{\prime \prime}\right)\right)^{\frac{1}{2}}\right. \\
+ & \left.\left(c 2^{k(d-1)}\right)^{\frac{1}{2}} 2^{-\frac{k}{2}(i+2) D} \mu\left(Q^{\prime \prime}\right)^{\frac{1}{2}}\right) \\
\leq & \frac{1}{3} C^{-\frac{1}{2}} 2^{-\frac{k}{2}(d-1-D)}\left(2^{\frac{k}{2}(d-D)} 2^{-\frac{k}{2}(i+1) D}\left(N \varepsilon_{0} \mu(Q)\right)^{\frac{1}{2}}\right. \\
+ & \left.C^{\frac{1}{2}} 2^{\frac{k}{2}(d-1-D)} 2^{-\frac{k}{2}(i+1) D} \mu\left(Q^{\prime \prime}\right)^{\frac{1}{2}}\right) \\
\leq & \frac{1}{3}\left(\varepsilon_{0} N\right)^{\frac{1}{2}} C^{-\frac{1}{2}} 2^{\frac{k}{2}} 2^{-\frac{k}{2}(i+1) D} \mu(Q)^{\frac{1}{2}}+\frac{1}{3} 2^{-\frac{k}{2}(i+1) D} \mu\left(Q^{\prime \prime}\right)^{\frac{1}{2}} .
\end{aligned}
$$


Combining (3.12) with (3.13), we continue using Lemma 3.3 and (3.9)

$$
\begin{aligned}
& \sum_{Q^{\prime \prime} \in \mathcal{Q}_{I}} \sum_{Q^{\prime} \prec Q^{\prime \prime}} \beta\left(Q^{\prime \prime}\right) \beta\left(Q^{\prime}\right) r_{Q^{\prime}}^{\frac{D}{2}} \mu\left(Q^{\prime}\right)^{\frac{1}{2}} \\
& \leq \frac{1}{3} C^{-\frac{1}{2}} 2^{-\frac{k}{2}(d-1-D)} \sum_{Q^{\prime \prime} \in \mathcal{Q}_{I}}\left(\sum_{\substack{Q^{\prime} \prec Q^{\prime \prime} \\
Q^{\prime} \cap Q_{p o r}^{\prime \prime}=\emptyset}} \beta\left(Q^{\prime}\right) r_{Q^{\prime}}^{\frac{D}{2}} \mu\left(Q^{\prime}\right)^{\frac{1}{2}}\right. \\
& \left.+\sum_{\substack{Q^{\prime} \prec Q^{\prime \prime} \\
Q^{\prime} \subset Q_{p o r}^{\prime \prime}}} \beta\left(Q^{\prime}\right) r_{Q^{\prime}}^{\frac{D}{2}} \mu\left(Q^{\prime}\right)^{\frac{1}{2}}\right) \\
& \leq \frac{1}{3} C^{-\frac{1}{2}} 2^{-\frac{k}{2}(d-1-D)} \sum_{Q^{\prime \prime} \in \mathcal{Q}_{I}}\left(\frac{1}{3}\left(\varepsilon_{0} N\right)^{\frac{1}{2}} C^{-\frac{1}{2}} 2^{\frac{k}{2}} 2^{-\frac{k}{2}(i+1) D} \mu(Q)^{\frac{1}{2}}\right. \\
& \left.+\frac{2}{3} 2^{-\frac{k}{2}(i+1) D} \mu\left(Q^{\prime \prime}\right)^{\frac{1}{2}}\right) \\
& \leq R\left(\varepsilon_{0}\right) r_{Q}^{\frac{D}{2}} \mu(Q)^{\frac{1}{2}}+\frac{2}{9} C^{-\frac{1}{2}} 2^{-\frac{k}{2}(d-1-D)} \sum_{Q^{\prime \prime} \in \mathcal{Q}_{I}} 2^{-\frac{k}{2}(i+1) D} \mu\left(Q^{\prime \prime}\right)^{\frac{1}{2}} \\
& \leq R\left(\varepsilon_{0}\right) r_{Q}^{\frac{D}{2}} \mu(Q)^{\frac{1}{2}}+\frac{2}{9} C^{-\frac{1}{2}} 2^{-\frac{k}{2}(d-1-D)} 2^{\frac{k d}{2}} 2^{-\frac{k}{2}(i+1) D} \mu(Q)^{\frac{1}{2}} \\
& \leq\left(R\left(\varepsilon_{0}\right)+\frac{2}{9} C^{-\frac{1}{2}} 2^{\frac{k}{2}}\right) r_{Q}^{\frac{D}{2}} \mu(Q)^{\frac{1}{2}} \\
& =\frac{1}{2} C^{-\frac{1}{2}} 2^{\frac{k}{2}} r_{Q}^{\frac{D}{2}} \mu(Q)^{\frac{1}{2}} \text {. }
\end{aligned}
$$

Recalling that $C \geq 2 d 2^{l}$, it is evident that $\# \mathcal{Q}_{B} \leq C 2^{k(d-1)}$. From (3.9) and Lemma 3.3 we obtain

$$
\begin{aligned}
& \sum_{Q^{\prime \prime} \in \mathcal{Q}_{B}} \sum_{Q^{\prime} \prec Q^{\prime \prime}} \beta\left(Q^{\prime}\right) \beta\left(Q^{\prime \prime}\right) r_{Q^{\prime}}^{\frac{D}{2}} \mu\left(Q^{\prime}\right)^{\frac{1}{2}} \\
& \leq \frac{1}{9} C^{-1} 2^{-k(d-1-D)}\left(C 2^{2 k\left(d-\frac{1}{2}\right)}\right)^{\frac{1}{2}} 2^{-\frac{k}{2}(i+2) D} \mu(Q)^{\frac{1}{2}} \\
& =\frac{1}{9} C^{-\frac{1}{2}} 2^{\frac{k}{2}} r_{Q}^{\frac{D}{2}} \mu(Q)^{\frac{1}{2}} .
\end{aligned}
$$

Putting together the above estimates, proves (3.10) when $n=2$.

Next we assume that (3.10) holds when $n=m$ and verify it in the case $n=m+1$. Again, we divide $Q$ into two regions of subcubes $\mathcal{Q}_{B}, \mathcal{Q}_{I} \subset \mathcal{Q}^{i+1}$ defined above. Lemma 3.5 is used to evaluate the sum in the interior region $\mathcal{Q}_{I}$ whereas the induction hypothesis is applied in the boundary region $\mathcal{Q}_{B}$. Let $R(\varepsilon)=R(\varepsilon, m+1)$ and $\varepsilon_{0}$ be as in (3.6) and (3.7). 
In $\mathcal{Q}_{I}$ we begin with

$$
\begin{aligned}
& \sum_{Q^{\prime \prime \prime} \in \mathcal{Q}_{I}} \sum_{Q^{\prime} \prec m}\left(\prod_{Q^{\prime \prime \prime}}^{m+1} \beta\left(Q_{i+j}^{\prime}\right)\right) r_{Q^{\prime}}^{\frac{D}{2}} \mu\left(Q^{\prime}\right)^{\frac{1}{2}} \\
= & \sum_{Q^{\prime \prime \prime} \in \mathcal{Q}_{I}} \sum_{Q^{\prime \prime} \prec_{m-1} Q^{\prime \prime \prime}} \prod_{j=1}^{m} \beta\left(Q_{i+j}^{\prime \prime}\right) \sum_{Q^{\prime} \prec Q^{\prime \prime}} \beta\left(Q^{\prime}\right) r_{Q^{\prime}}^{\frac{D}{2}} \mu\left(Q^{\prime}\right)^{\frac{1}{2}} .
\end{aligned}
$$

From (3.12) and (3.13) we get for the inner sum

$$
\begin{aligned}
& \sum_{Q^{\prime} \prec Q^{\prime \prime}} \beta\left(Q^{\prime}\right) r_{Q^{\prime}}^{\frac{D}{2}} \mu\left(Q^{\prime}\right)^{\frac{1}{2}} \\
& \leq \frac{1}{3}\left(\varepsilon_{0} N\right)^{\frac{1}{2}} C^{-\frac{1}{2}} 2^{\frac{k}{2}} 2^{-\frac{k m D}{2}} r_{Q}^{\frac{D}{2}} \mu(Q)^{\frac{1}{2}}+\frac{2}{3} r_{Q^{\prime \prime}}^{\frac{D}{2}} \mu\left(Q^{\prime \prime}\right)^{\frac{1}{2}} .
\end{aligned}
$$

Repeating this $m$ times and using (3.7), (3.9) and Lemma 3.3 yields

$$
\begin{aligned}
& \sum_{Q^{\prime \prime \prime} \in \mathcal{Q}_{I}} \sum_{Q^{\prime} \prec_{m} Q^{\prime \prime \prime}}\left(\prod_{j=1}^{m+1} \beta\left(Q_{i+j}^{\prime}\right)\right) r_{Q^{\prime}}^{\frac{D}{2}} \mu\left(Q^{\prime}\right)^{\frac{1}{2}} \\
& \leq R\left(\varepsilon_{0}\right) r_{Q}^{\frac{D}{2}} \mu(Q)^{\frac{1}{2}}+\left(\frac{2}{3}\right)^{m} \sum_{Q^{\prime \prime \prime} \in \mathcal{Q}_{I}} \beta\left(Q^{\prime \prime \prime}\right) r_{Q^{\prime \prime \prime}}^{\frac{D}{2}} \mu\left(Q^{\prime \prime \prime}\right)^{\frac{1}{2}} \\
& \leq R\left(\varepsilon_{0}\right) r_{Q}^{\frac{D}{2}} \mu(Q)^{\frac{1}{2}} \\
& +\frac{1}{3}\left(\frac{2}{3}\right)^{m} C^{-\frac{1}{2}} 2^{-\frac{k}{2}(d-1-D)} 2^{\frac{k d}{2}} 2^{-\frac{k}{2}(i+1) D} \mu(Q)^{\frac{1}{2}} \\
& \leq\left(R\left(\varepsilon_{0}\right)+\frac{1}{3}\left(\frac{2}{3}\right)^{m} C^{-\frac{1}{2}} 2^{\frac{k}{2}}\right) r_{Q}^{\frac{D}{2}} \mu(Q)^{\frac{1}{2}} \\
& \leq \frac{1}{2} C^{-\frac{1}{2}} 2^{\frac{k}{2}} r_{Q}^{\frac{D}{2}} \mu(Q)^{\frac{1}{2}} .
\end{aligned}
$$

Notice that $R\left(\varepsilon_{0}, i\right)<R\left(\varepsilon_{0}, m+1\right)$ for all $i=2, \ldots, m$ by (3.7).

Finally, the induction hypothesis, Lemma 3.3 and (3.9) combine to give in the boundary region $\mathcal{Q}_{B}$ (recall (3.4) $)$

$$
\begin{aligned}
& \sum_{Q^{\prime \prime} \in \mathcal{Q}_{B}} \sum_{Q^{\prime} \prec m}\left(\prod_{j=1}^{m+1} \beta\left(Q_{i+j}^{\prime}\right)\right) r_{Q^{\prime}}^{\frac{D}{2}} \mu\left(Q^{\prime}\right)^{\frac{1}{2}} \\
& \leq C^{-\frac{1}{2}} 2^{\frac{k}{2}} \sum_{Q^{\prime \prime} \in \mathcal{Q}_{B}} \beta\left(Q^{\prime \prime}\right) r_{Q^{\prime \prime}}^{\frac{D}{2}} \mu\left(Q^{\prime \prime}\right)^{\frac{1}{2}} \\
& \leq \frac{1}{3} C^{-1} 2^{\frac{k}{2}} 2^{-\frac{k}{2}(d-1-D)}\left(C 2^{k(d-1)}\right)^{\frac{1}{2}} 2^{-\frac{k}{2}(i+1) D} \mu(Q)^{\frac{1}{2}} \\
& =\frac{1}{3} C^{-\frac{1}{2}} 2^{\frac{k}{2}} r_{Q}^{\frac{D}{2}} \mu(Q)^{\frac{1}{2}} .
\end{aligned}
$$

The claim follows by summing up (3.14) and (3.15). 
In the previous lemma $p$ played no role. Our next result quantifies the fact that we really gain something if a large proportion of scales is porous.

Lemma 3.7. Let $0<p<1$ and

$$
D>d-p+\frac{\log (9 C)}{k \log 2} .
$$

Suppose that $\mu$ is a Radon measure on $\mathbb{R}^{d}$. Then there are $n \in \mathbb{N}$ and $K>1$ such that

$$
\prod_{j=0}^{L-1} C^{\frac{1}{2}} 2^{-\frac{k}{2}} \prod_{i=j n+1}^{(j+1) n} \beta\left(Q_{x}^{i}\right) \geq K^{L}
$$

for all $L \in \mathbb{N}$ and $x \in \mathbb{R}^{d}$ with

$$
\#\left\{0 \leq j \leq n L-1: \operatorname{por}\left(\mu, x, 2^{-k j+l}, \varepsilon\right) \geq \alpha\right\} \geq p n L
$$

for some $\varepsilon>0$. Here $n$ and $K$ are independent of $x$ and $L$.

Proof. Let

$$
D_{0}=d-p+\frac{\log (9 C)}{k \log 2} \text { and } \delta=\left(\frac{1}{D_{0}}-\frac{1}{D}\right)(d-p)>0 .
$$

Choose $n \in \mathbb{N}$ such that

$$
2^{k \delta D n}>C^{-1} 2^{k}
$$

Obviously, $Q_{x}^{j}$ is porous whenever $\operatorname{por}\left(\mu, x, 2^{-k(j-1)+l}, \varepsilon\right) \geq \alpha$, and thus

$$
\#\left\{1 \leq j \leq n L: Q_{x}^{j} \text { is porous }\right\} \geq p n L .
$$

By (3.5) we have

$$
\begin{aligned}
& \prod_{j=0}^{L-1} C^{\frac{1}{2}} 2^{-\frac{k}{2}} \prod_{i=j n+1}^{(j+1) n} \beta\left(Q_{x}^{i}\right) \\
& =\left(C^{\frac{1}{2}} 2^{-\frac{k}{2}}\right)^{L} \prod_{i=1}^{L n} \beta\left(Q_{x}^{i}\right) \\
& \geq\left(C^{\frac{1}{2}} 2^{-\frac{k}{2}}\right)^{L}\left(\frac{1}{3}\right)^{L n}\left(C^{-\frac{1}{2}} 2^{-\frac{k}{2}(d-1-D)}\right)^{p L n}\left(2^{-\frac{k}{2}(d-D)}\right)^{(1-p) L n} \\
& =\left(\left(C^{\frac{1}{D}} 2^{-\frac{k}{D}}\right)^{L}\left(3^{-\frac{2}{D}} C^{-\frac{p}{D}} 2^{-k\left(\frac{d-p}{D}-1\right)}\right)^{L n}\right)^{\frac{D}{2}} \\
& \geq\left(\left(C^{\frac{1}{D}} 2^{-\frac{k}{D}}\right)^{L} 2^{k \delta L n}\left((9 C)^{-\frac{1}{D_{0}}} 2^{-k\left(\frac{d-p}{D_{0}}-1\right)}\right)^{L n}\right)^{\frac{D}{2}} \\
& =\left(C 2^{-k} 2^{k \delta D n}\right)^{\frac{L}{2}}
\end{aligned}
$$

which gives the claim by (3.16). 
Remark 3.8. Mean porosity is defined in terms of scales $2^{-i}$ where $i \in \mathbb{N}$. However, in the proof of Theorem 3.1 we need to use scales of the form $2^{-k i+m}$ where $k, m \in \mathbb{N}$. This problem may be overcome by the following observation: Let $\varepsilon>0$ and $x \in \mathbb{R}^{n}$ such that $\#\left\{1 \leq j \leq N k: \operatorname{por}\left(\mu, x, 2^{-j}, \varepsilon\right) \geq \alpha\right\} \geq p N k$. Then there is an integer $t$ with $0 \leq t \leq k-1$ such that

$$
\#\left\{1 \leq j \leq N: \operatorname{por}\left(\mu, x, 2^{-k j+t}, \varepsilon\right) \geq \alpha\right\} \geq p N \text {. }
$$

Observe that the starting scale plays no role in Lemmas 3.6 and 3.7 and we choose it (out of $k$ possibilities) depending on the point.

Proof of Theorem 3.1. Suppose that $\mu$ is mean $(\alpha, p)$-porous for $\frac{15}{32}<\alpha<\frac{1}{2}$ for which (3.8) holds and for $0 \leq p \leq 1$. It is clearly enough to prove the claim for such $\alpha$ 's. We shall prove that

$$
\operatorname{dim}_{\mathrm{p}} \mu \leq d-p+\frac{\log (9 C)}{k \log 2}
$$

where $C=C(d)$ and $k=k(\alpha)$ are as in Lemma 3.6. This implies the claim since, by (3.1),

$$
\frac{\log (9 C)}{k \log 2} \leq \frac{C^{\prime}(d)}{\log \left(\frac{1}{1-2 \alpha}\right)}
$$

We may assume without loss of generality that $\mu\left(\mathbb{R}^{d}\right)=1$ and $\operatorname{spt} \mu \subset[0,1]^{d}$.

Let $0<p^{\prime}<p, D>d-p^{\prime}+\frac{\log (9 C)}{k \log 2}$ and $K$ and $n$ be as in Lemma 3.7. Take $\varepsilon_{0}$ as in (3.6) with $R(\varepsilon)=R(\varepsilon, n)$. Since $\mu$ is mean $(\alpha, p)$-porous there are a Borel set $B \subset \mathbb{R}^{d}$ and constants $\eta>0,0<\varepsilon<\varepsilon_{0}$ and $I_{0} \in \mathbb{N}$ such that $\mu(B)>\eta$ and

$$
\#\left\{1 \leq j \leq I: \operatorname{por}\left(\mu, x, 2^{-j}, \varepsilon\right) \geq \alpha\right\}>p^{\prime} I
$$

for all $I \geq I_{0}$ and for all $x \in B$. Let $N_{0}$ be so large that

$$
K^{N_{0}}>\frac{4 k}{\eta} \text { and } N_{0} k n \geq I_{0}
$$

Let $\mathcal{Q}$ be any finite collection of $2^{k n}$-adic cubes with $r_{Q}=2^{-N_{Q} k n} \leq 2^{-N_{0} k n}$ for all $Q \in \mathcal{Q}$. For those $Q \in \mathcal{Q}$ for which $Q \cap B \neq \emptyset$, we define $\tau(Q)=\frac{D}{2}$. By Remark 3.8 there are $t=t(Q) \in\{0, \ldots, k-1\}$ and $x \in Q$ such that

$$
\#\left\{1 \leq j \leq N_{Q} n: \operatorname{por}\left(\mu, x, 2^{-k j+t+l}, \varepsilon\right) \geq \alpha\right\} \geq p^{\prime} N_{Q} n
$$

provided that $Q \cap B \neq \emptyset$. (Note that $l$ is added here because it appears in the assumptions of Lemma 3.7 via the way we defined porous cubes in the chapter after (3.1).) For each such $Q \in \mathcal{Q}$ fix $Q^{\prime}$ with $Q \subset Q^{\prime}$ and $r_{Q^{\prime}}=2^{-N_{Q} k n+t}$. In this way we obtain $k$ collections $\mathcal{Q}_{m}$ of $2^{k n}$-adic cubes scaled by the factor $2^{m}$, $m=0, \ldots, k-1$. Since $N_{Q} \geq N_{0}$ we have by Lemma 3.7 and (3.18)

$$
r_{Q}^{\tau(Q)} \mu(Q)^{1-\frac{\tau(Q)}{D}}<\frac{\eta}{4 k} \prod_{j=0}^{N_{Q}-1} C^{\frac{1}{2}} 2^{-\frac{k}{2}} \prod_{i=j n+1}^{(j+1) n} \beta\left(Q_{i}\right) r_{Q}^{\frac{D}{2}} \mu(Q)^{\frac{1}{2}}
$$


for each $Q \in \mathcal{Q}_{m}$ where $\beta\left(Q_{i}\right)$ is as in (3.5). Summing over all $Q$ 's and using (3.11), we get for all $m=0, \ldots, k-1$

$$
\begin{aligned}
& \sum_{\substack{Q \in \mathcal{Q}_{m} \\
Q \cap B \neq \emptyset}} r_{Q}^{\tau(Q)} \mu(Q)^{1-\frac{\tau(Q)}{D}} \\
& <\frac{\eta}{4 k} \sum_{\substack{Q \in \mathcal{Q}_{m} \\
Q \cap B \neq \emptyset}} \prod_{j=0}^{N_{Q}-1} C^{\frac{1}{2}} 2^{-\frac{k}{2}} \prod_{i=j n+1}^{(j+1) n} \beta\left(Q_{i}\right) r_{Q}^{\frac{D}{2}} \mu(Q)^{\frac{1}{2}} \\
& \leq \frac{\eta}{4 k} r_{[0,1]^{d}}^{\frac{D}{2}} \mu\left(\mathbb{R}^{d}\right)^{\frac{1}{2}}=\frac{\eta}{4 k} .
\end{aligned}
$$

Since $r_{Q}^{\frac{D}{2}} \mu(Q)^{\frac{1}{2}} \leq r_{Q^{\prime}}^{\frac{D}{2}} \mu\left(Q^{\prime}\right)^{\frac{1}{2}}$, inequality (3.19) implies that

$$
\sum_{\substack{Q \in \mathcal{Q} \\ Q \cap B \neq \emptyset}} r_{Q}^{\tau(Q)} \mu(Q)^{1-\frac{\tau(Q)}{D}} \leq \sum_{m=0}^{k-1} \sum_{\substack{Q \in \mathcal{Q}_{m} \\ Q \cap B \neq \emptyset}} r_{Q}^{\tau(Q)} \mu(Q)^{1-\frac{\tau(Q)}{D}} \leq k \frac{\eta}{4 k}=\frac{\eta}{4}
$$

Suppose that $Q \in \mathcal{Q}$ with $Q \cap B=\emptyset$ and choose $\tau(Q)>0$ so small that $r_{Q}^{\tau(Q)} \mu(Q)^{1-\frac{\tau(Q)}{D}} \leq \frac{1-\frac{\eta}{2}}{1-\eta} \mu(Q)$. This choice is possible since $r_{Q}^{\tau} \mu(Q)^{1-\frac{\tau}{D}} \rightarrow \mu(Q)$ as $\tau \downarrow 0$. Now

$$
\sum_{\substack{Q \in \mathcal{Q} \\ Q \cap B=\emptyset}} r_{Q}^{\tau(Q)} \mu(Q)^{1-\frac{\tau(Q)}{D}} \leq \frac{1-\frac{\eta}{2}}{1-\eta} \mu\left(\mathbb{R}^{d} \backslash B\right)<1-\frac{\eta}{2} .
$$

Combining (3.20) and (3.21) gives

$$
\sum_{Q \in \mathcal{Q}} r_{Q}^{\tau(Q)} \mu(Q)^{1-\frac{\tau(Q)}{D}}<1-\frac{\eta}{4}<\mu\left(\mathbb{R}^{d}\right) .
$$

Since the same upper bound is valid also for infinite collections of cubes Lemma 3.2 implies that $\operatorname{dim}_{\mathrm{p}} \mu \leq D$. Letting $D \downarrow d-p+\frac{\log (9 C)}{k \log 2}$ completes the proof of (3.17).

The following example shows that we cannot get a better upper estimate on the dimension than

$$
d-p+p \frac{C}{\log \left(\frac{1}{1-2 \alpha}\right)}
$$

even for mean porous sets. For simplicity, we consider the case $d=1$. We use the notation $\operatorname{dim}_{H}$ for Hausdorff dimension. The idea of the example is the following: First we take $m n l$ steps in the construction of a standard Cantor set where each interval is substituted by two subintervals of length $2^{-n k}$. This will give us $(m n l-1) n k$ scales where porosity is at least $\frac{1}{2}-2^{-k n}$. Next we divide each construction interval into $2^{(n k-m n) n k l}$ subintervals and choose every other of them. This guarantees that the dimension will be large enough but the cost of 
it is the term $-n k$ in the number of porous scales. To get rid of this we iterate this procedure increasing $l$ at every step.

Example 3.9. Fix $\left.p=\frac{m}{k} \in\right] 0,1[\cap \mathbb{Q}$. Define for all $n \in \mathbb{N}$ a Cantor-type meanporous set

$$
A_{p, n}=\bigcap_{j=1}^{\infty} \bigcup_{\substack{g_{i} \in \mathcal{S}_{i} \\ i=1, \ldots, j}}\left(g_{1} \circ \cdots \circ g_{j}\right)([0,1])
$$

with

$$
\mathcal{S}_{l}=\left\{K_{j_{1}} \circ \cdots \circ K_{j_{m n l}} \circ f_{i}: i \in\left\{0, \ldots, 2^{(n k-m n) n k l-1}-1\right\}, j_{t} \in\{1,2\} \forall t\right\},
$$

where

$$
\begin{aligned}
f_{i} & : \mathbb{R} \rightarrow \mathbb{R}: x \mapsto 2^{-(n k-m n) n k l} x+i 2^{-(n k-m n) n k l+1}, \\
K_{1}: \mathbb{R} & \rightarrow \mathbb{R}: x \mapsto 2^{-n k} x \text { and } \\
K_{2}: & \rightarrow \mathbb{R}: x \mapsto 2^{-n k} x+1-2^{-n k} .
\end{aligned}
$$

A standard calculation shows that

$$
\operatorname{dim}_{\mathrm{H}} A_{p, n}=1-\frac{m}{k}+\frac{m}{n k^{2}}
$$

On scales $2^{-s}$, where

$$
s-\frac{l^{2}+l}{2}(n k)^{2} \in\{0, \ldots,(m n l-1) n k-1\} \text { for some } l \in \mathbb{N}
$$

the porosity at each point $x \in A_{p, n}$ is at least $\frac{1}{2}-2^{-k n}$. Hence, $A_{p, n}$ is mean $\left(\frac{1}{2}-2^{-k n}, p\right)$-porous. Finally, we notice that

$$
1-p+p \frac{\frac{1}{2} \log 2}{\log \left(\frac{1}{1-2 \alpha}\right)}=1-\frac{m}{k}+\frac{m}{2 k(k n-1)} \leq \operatorname{dim}_{\mathrm{H}} A_{p, n} .
$$

\section{Mean porous measures are not neCessarily approximable by MEAN POROUS SETS}

The aim of this section is to clarify relations between mean porosities of sets and those of measures by verifying that, contrary to [BS, Proposition 1], mean porous measures cannot be approximated by mean porous sets. For simplicity, we restrict our consideration to $\mathbb{R}$. Our goal is to construct a mean porous measure $\mu$ on $[0,1]$ with the property that any mean porous set has zero $\mu$-measure.

Intuitively, the measure $\mu$ is constructed in the following way: For all positive integers $i$, we define slowly decaying weights $w(i)$ and start with the uniform measure on the unit interval. On $i^{\text {th }}$ step of the construction we redistribute the measure on the dyadic intervals of length $2^{-i}$ by attaching weight $w(i)$ to one half of the dyadic interval of length $2^{-(i-1)}$ and $1-w(i)$ to the other half. We 
alternate the half of the interval which gets most of the measure. To be precise, define for all $0<p \leq 1$

$$
\begin{array}{r}
\beta_{p}(\mu)=\sup \{\alpha \geq 0: \mu(A)>0 \text { for some mean } \\
(\alpha, p) \text {-porous set } A \subset \mathbb{R}\}
\end{array}
$$

and proceed by modifying the construction of [EJJ, Example 4]: For positive integers $i$, set $w(i)=\frac{1}{\log (i+2)}$ and

$$
s(i)= \begin{cases}w(i) & \text { if } i \text { is odd } \\ 1-w(i) & \text { if } i \text { is even. }\end{cases}
$$

For all binary sequences $j_{1} \ldots j_{i}$, we denote by $I_{j_{1} \ldots j_{i}}$ the closed dyadic interval of length $2^{-i}$ whose left endpoint in binary representation is $0, j_{1} j_{2} \ldots j_{i}$. Let $\mu$ be the unique Radon probability measure on $[0,1]$ determined by the formula

$$
\mu\left(I_{j_{1} \ldots j_{i}}\right)=\prod_{k=1}^{i}(1-s(k))^{j_{k}} s(k)^{1-j_{k}}
$$

for all binary sequences $j_{1} \ldots j_{i}$. We change continuously the side of the larger weight since we want to avoid the technical difficulties caused by the fact that at some scales the small ball giving the porosity might be outside the dyadic interval containing the point we are considering.

Theorem 4.1. The measure $\mu$ has the following properties:

(1) $\beta_{p}(\mu)=0$ for all $0<p \leq 1$,

(2) $\mu$ is $\frac{1}{8}$-porous and

(3) $\mu$ is mean $(\alpha, 1)$-porous for all $0 \leq \alpha<\frac{1}{2}$.

Remark 4.2. For (1) we need to take slowly decreasing weights $w(i)$ whilst for (2) and (3) we only need that $w(i) \rightarrow 0$ as $i \rightarrow \infty$. Note that in (2) the value of porosity is not optimal. Our aim is only to demonstrate that even porous measures need not be approximable by mean porous sets.

Theorem 4.1 will be proven as a consequence of several lemmas. The first one, Lemma 4.3, is a corollary of the strong law of large numbers. For $x \in[0,1]$, let $x_{i}$ denote the $i$ :th digit of $x$ in the binary representation. The non-uniqueness of the representation plays no role here since $\mu$ is easily seen to be non-atomic.

Lemma 4.3. For $\mu$-almost all $x \in[0,1]$, we have

$$
\lim _{i \rightarrow \infty} \frac{\#\left\{1 \leq j \leq i: x_{j}=x_{j+1}\right\}}{i}=0 .
$$

Proof. Applying the strong law of large numbers, see [Fe, Chapter X.7] to the sequence of mutually independent random variables $X_{i}=x_{i}$, if $i$ is odd, and 
$X_{i}=1-x_{i}$, if $i$ is even, gives

$$
\lim _{i \rightarrow \infty} \frac{\#\left\{1 \leq j \leq i: x_{j}=q(j)\right\}}{i}=1
$$

for $\mu$-almost all $x \in[0,1]$. Here $q(j)=j \bmod 2$. From this the claim follows easily.

For all positive integers $j$, we use the notation $\mathcal{D}^{j}$ for the half-open dyadic subintervals of $[0,1]$ having length $2^{-j}$. (We do not use the notation $\mathcal{Q}^{j}$ introduced in Section 3 since we want to distinguish between powers of 2 and $2^{k}$.) Letting $m$ be a positive integer and $E \subset[0,1]$, we say that $D \in \mathcal{D}^{j}$ contains an $m$-hole of $E$ if there is $D^{\prime} \in \mathcal{D}^{j+m}$ such that $D^{\prime} \subset D$ and $D^{\prime} \cap E=\emptyset$.

Lemma 4.4. Suppose that $E \subset[0,1]$ is mean $(\alpha, p)$-porous for some $\alpha>0$ and $0<p \leq 1$. Let $m$ be a positive integer such that $2^{-m}<\frac{\alpha}{4}$. For $x \in[0,1]$, let $D_{x}^{j} \in \mathcal{D}^{j}$ be such that $\{x\}=\bigcap_{j=1}^{\infty} D_{x}^{j}$. Then for $\mu$-almost all $x \in[0,1]$, we have

$$
\liminf _{i \rightarrow \infty} \frac{\#\left\{1 \leq j \leq i: D_{x}^{j} \text { contains an } m \text {-hole of } E\right\}}{i}>\frac{p}{8} \text {. }
$$

Proof. Observe that for all $x \in E$ there exists $N_{1}$ such that

$$
\#\left\{1 \leq j \leq i: \operatorname{por}\left(E, x, 2^{-j}\right) \geq \alpha\right\}>\frac{p i}{2}
$$

for all $i>N_{1}$. Lemma 4.3 implies that for $\mu$-almost every $x \in[0,1]$ we may choose a positive integer $N_{2}$ with $N_{2}>\frac{16}{p}$ such that

$$
\#\left\{1 \leq j \leq i: x_{j}=x_{j+1}\right\}<\frac{p i}{4}
$$

for all $i>N_{2}$. Hence, for $\mu$-almost every $x \in[0,1]$

$$
\#\left\{3 \leq j \leq i: \operatorname{por}\left(E, x, 2^{-j}\right) \geq \alpha \text { and } x_{j-1} \neq x_{j}\right\}>\frac{p i}{8}
$$

for all $i>\max \left\{N_{1}, N_{2}\right\}$.

Consider $x \in[0,1]$ for which (4.1) holds and a positive integer $j \geq 3$ such that $x_{j-1} \neq x_{j}$ and $E$ is $\alpha$-porous for scale $j$ at $x$. An easy calculation yields that ]$x-2^{-j}, x+2^{-j}\left[\subset D_{x}^{j-2}\right.$. Since, by the choice of $m$, there is $D \in \mathcal{D}^{j+m-2}$ such that $D \cap E=\emptyset$ and $D \subset] x-2^{-j}, x+2^{-j}\left[\right.$, it follows that $D_{x}^{j-2}$ contains an $m$-hole of $E$. Now (4.1) gives the claim.

For the remaining two lemmas we need the following notation: Let $l$ and $m$ be positive integers and $E \subset[0,1]$. If $Q \in \mathcal{D}^{(j+1)(l+m)}$ and $Q^{\prime} \in \mathcal{D}^{j(l+m)}$ such that $Q \subset Q^{\prime}$, we use the same notation $Q \prec Q^{\prime}$ as in Section 3 with $k$ replaced by $l+m$. We say that $Q^{\prime} \in \mathcal{D}^{j(l+m)}$ is porous with respect to $E$ if $Q \cap E=\emptyset$ for some $Q \prec Q^{\prime}$. For $Q \in \mathcal{D}^{i(l+m)}$ and $j=0, \ldots, i$, let $Q_{j}$ be the unique interval in $\mathcal{D}^{j(l+m)}$ for which $Q \subset Q_{j}$. Obviously, these definitions depend on the indices $l$ and $m$, but in what follows this dependence does not cause any confusion and 
it is therefore omitted for the sake of simplicity. We refer by $Q$ to $2^{l+m}$-adic intervals and by $D$ to dyadic intervals. In particular for $D \in \mathcal{D}^{i}$ there exists unique intervals $D_{j} \in \mathcal{D}^{j}$ for all $j=0, \ldots, i$.

Our next lemma is a consequence of the previous one.

Lemma 4.5. Suppose that $A \subset[0,1]$ is mean $(\alpha, p)$-porous for some $\alpha>0$ and $0<p \leq 1$ and $\mu(A)>0$. Let $m$ be a positive integer with $2^{-m}<\frac{\alpha}{4}$. Then there are $p^{\prime}>0$, positive integers $l$ and $N_{0}$ and $E \subset A$ with $\mu(E)>0$ such that for all positive integers $i \geq N_{0}$ and for all $Q \in \mathcal{D}^{i(l+m)}$ we have

$$
\#\left\{0 \leq j \leq i-1: Q_{j} \text { is porous with respect to } E\right\} \geq p^{\prime} i \text {. }
$$

Proof. Lemma 4.4 implies the existence of $E \subset A$ with $\mu(E)>0$ and that of a positive integer $N$ such that for all positive integers $i \geq N$ and for all $D \in \mathcal{D}_{i}$ with $D \cap E \neq \emptyset$ we have

$$
\#\left\{1 \leq j \leq i: D_{j} \text { contains an } m \text {-hole of } E\right\}>\frac{p i}{8} \text {. }
$$

It follows that (4.2) is valid for all $D \in \mathcal{D}^{i}$ when $i>N /\left(1-\frac{p}{8}\right)$. To see this, let $D \in \mathcal{D}^{i}$. Denote by $j_{0}$ the largest $j$ with $E \cap D_{j} \neq \emptyset$. If $j_{0} \geq N$, then the claim clearly holds by (4.2). On the other hand, assuming that $j_{0}<N$, we obtain

$$
\left\{1 \leq j \leq i: D_{j} \text { contains an } m \text {-hole of } E\right\} \geq i-j_{0} \geq i-N>\frac{p i}{8}
$$

by the choice of $i$.

Choose a positive integer $l>N /\left(1-\frac{p}{8}\right)$ so large that $m<\frac{p}{16}(m+l)$. Dividing the sequence $1,2, \ldots, i(m+l)$ into successive blocks of length $m+l$, it follows that for all positive integers $i$ and for all $D \in \mathcal{D}^{i(m+l)}$ we have

$$
\begin{aligned}
& \#\left\{1 \leq j \leq i: D_{k} \text { contains an } m \text {-hole of } E\right. \text { for some } \\
& \qquad(j-1)(m+l) \leq k \leq j(m+l)-m\}>\frac{p i}{16} .
\end{aligned}
$$

Indeed, if this is not the case, then

$$
\begin{gathered}
\#\left\{1 \leq k \leq i(m+l): D_{k} \text { contains an } m \text {-hole of } E\right\} \\
\leq \frac{p i}{16} l+i m \leq \frac{p i}{8}(m+l)
\end{gathered}
$$

giving a contradiction to (4.2). But if $D_{k}$ contains an $m$-hole of $E$ for some $(j-1)(m+l) \leq k \leq j(m+l)-m$, then $D_{(j-1)(m+l)}$ is porous with respect to $E$, and we obtain

$$
\#\left\{0 \leq j \leq i-1: Q_{j} \text { is porous with respect to } E\right\}>\frac{p i}{16}
$$

for all $Q \in \mathcal{D}^{i(m+l)}$.

Remark 4.6. The use of $l$ and $m$ prevents us from counting same holes twice. 
Next we introduce for each interval $Q$ a weight $\eta(Q)$ which is equal to 1 if $Q$ is not porous and 1 minus the portion of the measure of the hole if $Q$ is porous. For this purpose, let $l$ and $m$ be positive integers and let $E \subset[0,1]$. Define

$$
\eta(Q)=\left\{\begin{array}{l}
1 \text { if } Q \text { is not porous w.r.t. } E \\
1-w(i(l+m)+1) \times \cdots \times w((i+1)(l+m)) \text { otherwise }
\end{array}\right.
$$

for all non-negative integers $i$ and $Q \in \mathcal{D}^{i(m+l)}$.

In formula (4.3) of Lemma 4.7 we prove that the product of weights of all predecessors of $Q$ converges uniformly to 0 as the size of $Q$ decreases. The reason for this is quite simple: on a positive percentage of scales there are porous intervals $Q$ with $\eta(Q)<1$ and the product converges to 0 since the measure of the holes decreases very slowly. On the other hand, the proof of formula (4.4) is based on the definition of $\eta$. It guarantees that we may utilize porous scales.

Lemma 4.7. Suppose that $A \subset[0,1]$ is mean $(\alpha, p)$-porous for some $\alpha>0$ and $0<p \leq 1$ and $\mu(A)>0$. Let $p^{\prime}>0, E \subset A$ and positive integers $m, l$ and $N_{0}$ be as in Lemma 4.5. Then for all positive integers $i \geq N_{0}$ and for all $Q \in \mathcal{D}^{i(m+l)}$ we have

$$
\prod_{j=0}^{i} \eta\left(Q_{j}\right) \leq c(i)
$$

where $c(i)$ is a constant depending only on $i, l, m$ and $p^{\prime}$ and $c(i) \rightarrow 0$ as $i \rightarrow \infty$. (The dependence on all other parameters but $i$ is irrelevant for our purposes.) Moreover,

$$
\sum_{\substack{Q \in \mathcal{D}^{i(m+l)} \\ Q \cap E \neq \emptyset}} \frac{\mu(Q)}{\prod_{j=0}^{i-1} \eta\left(Q_{j}\right)} \leq \mu([0,1])=1
$$

for all positive integers $i$.

Proof. By decreasing $p^{\prime}$, we may assume that $\frac{1}{p^{\prime}}$ is an integer such that $\frac{1}{p^{\prime}}>N_{0}$. (Note that in Lemma 4.5 the integer $N_{0}$ is independent of $p^{\prime}$.) Consider $i>\frac{1}{p^{\prime}}$. Letting $k$ be a positive integer with $\frac{k}{p^{\prime}}<i \leq \frac{k+1}{p^{\prime}}$ and applying Lemma 4.5 
successively for the indices $\frac{1}{p^{\prime}}, \frac{2}{p^{\prime}}, \ldots, \frac{k}{p^{\prime}}$, gives

$$
\begin{aligned}
& \prod_{j=0}^{i} \eta\left(Q_{j}\right) \leq \prod_{q=1}^{k}\left(1-w\left(\left(\frac{q}{p^{\prime}}-1\right)(l+m)+1\right) \times \cdots \times w\left(\frac{q}{p^{\prime}}(l+m)\right)\right) \\
& \quad=\exp \left(\sum_{q=1}^{k} \log \left(1-w\left(\left(\frac{q}{p^{\prime}}-1\right)(l+m)+1\right) \times \cdots \times w\left(\frac{q}{p^{\prime}}(l+m)\right)\right)\right) \\
& \quad<\exp \left(-\sum_{q=1}^{k} w\left(\left(\frac{q}{p^{\prime}}-1\right)(l+m)+1\right) \times \cdots \times w\left(\frac{q}{p^{\prime}}(l+m)\right)\right) \\
& \quad<\exp \left(-\sum_{q=1}^{k}\left(\log \left(\frac{q}{p^{\prime}}(l+m)+2\right)\right)^{-(l+m)}\right) \underset{k \rightarrow \infty}{\longrightarrow} 0
\end{aligned}
$$

implying (4.3).

Inequality (4.4) is valid since

$$
\begin{aligned}
\sum_{\substack{Q \in \mathcal{D}^{i(m+l)} \\
Q \cap E \neq \emptyset}} \frac{\mu(Q)}{\prod_{j=0}^{i-1} \eta\left(Q_{j}\right)} & =\sum_{\substack{Q^{\prime} \in \mathcal{D}^{(i-1)(m+l)} \\
Q^{\prime} \cap E \neq \emptyset}} \frac{1}{\prod_{j=0}^{i-1} \eta\left(Q_{j}^{\prime}\right)} \sum_{\substack{Q \prec Q^{\prime} \\
Q \cap E \neq \emptyset}} \mu(Q) \\
& \leq \sum_{\substack{Q^{\prime} \in \mathcal{D}^{(i-1)(m+l)} \\
Q^{\prime} \cap E \neq \emptyset}} \frac{\eta\left(Q^{\prime}\right) \mu\left(Q^{\prime}\right)}{\eta\left(Q^{\prime}\right) \prod_{j=0}^{i-2} \eta\left(Q_{j}^{\prime}\right)} \\
& =\sum_{\substack{Q^{\prime} \in \mathcal{D}^{(i-1)(m+l)} \\
Q^{\prime} \cap E \neq \emptyset}} \frac{\mu\left(Q^{\prime}\right)}{\prod_{j=0}^{i-2} \eta\left(Q_{j}^{\prime}\right)}
\end{aligned}
$$

Here the inequality follows by dividing the inner summation into the two possible cases: either $Q \cap E \neq \emptyset$ for all $Q \prec Q^{\prime}$ or $Q \cap E=\emptyset$ for some $Q \prec Q^{\prime}$.

Now we are ready to prove the main theorem of this section.

Proof of Theorem 4.1. Assume contrary to the claim (1) that there is $\alpha>0$ and a mean $(\alpha, p)$-porous set $A \subset[0,1]$ with $\mu(A)>0$. Using Lemmas 4.5 and4.7, we find positive integers $m$ and $l$ and $E \subset A$ with $\mu(E)>0$ satisfying the estimates (4.3) and (4.4). Taking $N_{0}$ as in Lemma 4.7, we get for all $i \geq N_{0}$

$$
\frac{\mu(E)}{c(i)} \leq \sum_{\substack{Q \in \mathcal{D}^{(i+1)(m+l)} \\ Q \cap E \neq \emptyset}} \frac{\mu(Q)}{\prod_{j=0}^{i} \eta\left(Q_{j}\right)} \leq 1,
$$

giving $\mu(E) \leq c(i) \rightarrow 0$ as $i \rightarrow \infty$ contrary to $\mu(E)>0$. This proves (1).

An elementary calculation proves (2), for details see [EJJ, Example 4]. Clearly, $\frac{1}{8}$ is not the best possible value, but this is irrelevant for our purposes. 
For the remaining part (3), given $0<\alpha<\frac{1}{2}$, fix a positive integer $n$ such that $2^{-n}<\frac{1}{2}-\alpha$. As in Lemma 4.3, we obtain

$$
\lim _{i \rightarrow \infty} \frac{\#\left\{1 \leq j \leq i: x_{k}=x_{k+1} \text { for some } j \leq k<j+n\right\}}{i}=0
$$

for $\mu$-almost all $x \in[0,1]$. Thus, for $0<p<1$, we find for $\mu$-almost all $x \in[0,1]$ a positive integer $N$ such that

$$
\left\{1 \leq j \leq i: x_{k}=x_{k+1} \text { for some } j \leq k<j+n\right\}<(1-p) i
$$

for all $i>N$. This implies that $\mu$ is mean $(\alpha, p)$-porous at $\mu$-almost all points $x \in[0,1]$ (for details, see [EJJ, Example 4]), and consequently (3) holds.

\section{REFERENCES}

[BS] D. B. Beliaev and S. K. Smirnov, On dimension of porous measures, Math. Ann. 323 (2002), 123-141.

[C] C. D. Cutler, Strong and weak duality principles for fractal dimension in Euclidean space, Math. Proc. Cambridge Philos. Soc. 118 (1995), 393-410.

[EJJ] J.-P. Eckmann, E. Järvenpää and M. Järvenpää, Porosities and dimensions of measures, Nonlinearity 13 (2000), 1-18.

[Fe] W. Feller, An Introduction to Probability Theory and Its Applications, John Wiley \& Sons, New York, 1950.

[JJ1] E. Järvenpää and M. Järvenpää, Porous measures on $\mathbb{R}^{n}$ : local structure and dimensional properties, Proc. Amer. Math. Soc (2) 130 (2002), 419-426.

[JJ2] E. Järvenpää and M. Järvenpää, Average homogeneity and dimensions of measures, Math. Ann. 331 (2005), 557-576.

[JJKS] E. Järvenpää, M. Järvenpää, A. Käenmäki and V. Suomala, Asymptotically sharp dimension estimates for $k$-porous sets, Math. Scand. 97 (2005), 309-318.

[KR] P. Koskela and S. Rohde, Hausdorff dimension and mean porosity, Math. Ann. 309 (1997), 593-609.

[Ma1] P. Mattila, Distribution of sets and measures along planes, J. London Math. Soc. (2) 38 (1988), 125-132.

[Ma2] P. Mattila, Geometry of Sets and Measures in Euclidean Spaces: Fractals and rectifiability, Cambridge University Press, Cambridge, 1995.

[S] A. Salli, On the Minkowski dimension of strongly porous fractal sets in $\mathbb{R}^{n}$, Proc. London Math. Soc. (3) 62 (1991), 353-372.

DB: Department of Mathematics, Princeton University, Fine Hall, Washington Road Princeton NJ 08544-1000, USA

EJ,MJ,AK,TR and VS: Department of Mathematics and Statistics, P.O. Box 35 (MAD), FIN-40014 University of Jyväskylä, Finland 
SS: Department of Mathematics, University of Geneva, 2-4 Rue du Lièvre, Case postale 64, 1211 Genève 4 , Switzerland

E-mail address: dbeliaev@math.princeton.edu

E-mail address: esaj@maths.jyu.fi

E-mail address: amj@maths.jyu.fi

E-mail address: antakae@maths.jyu.fi

E-mail address: tamaraja@maths.jyu.fi

E-mail address: Stanislav.Smirnov@math.unige.ch

E-mail address: visuomal@maths.jyu.fi 